\title{
Ultrafast electron emission from metallic nanotip arrays induced by near infrared femtosecond laser pulses
}

\author{
S. Tsujino, ${ }^{1, a)}$ P. Beaud, ${ }^{1}$ E. Kirk, ${ }^{1}$ T. Vogel, ${ }^{1}{ }^{1}$ H. Sehr, ${ }^{1}$ J. Gobrecht, ${ }^{1}$ and A. Wrulich ${ }^{2}$ \\ ${ }^{1}$ Department of Synchrotron Radiation and Nanotechnology, Paul Scherrer Institut, \\ CH-5232 Villigen-PSI, Switzerland \\ ${ }^{2}$ PSI XFEL, Paul Scherrer Institut, CH-5232 Villigen-PSI, Switzerland
}

(Received 2 April 2008; accepted 19 April 2008; published online 12 May 2008)

\begin{abstract}
The authors explore the impact of femtosecond light pulses on the field-emission properties of single-gate molybdenum field-emitter arrays with nanometer scale tip apex. Despite the small fraction of the emission area, we observed a single-photon photoelectric current from the emitter tips on top of the dc field-emission current under the irradiation of $50 \mathrm{fs}$ laser pulses at a wavelength of $800 \mathrm{~nm}$ with an external quantum efficiency up to $\sim 2 \times 10^{-7}$ and the emitter tip quantum efficiency of $\sim 10^{-2}$. The result indicates that metallic field-emitter arrays are promising for applications that require high-brightness short electron beams. (C) 2008 American Institute of Physics. [DOI: 10.1063/1.2924290]
\end{abstract}

Laser-induced photoemission in metallic needle cathodes with nanometer-scale sharpness has attracted attention to generate bright, low-emittance, and short electron bunches with durations down to the femtosecond range, ideal for applications such as the time-resolved electron microscopy, ${ }^{1}$ compact free-electron lasers, ${ }^{2}$ or scanning probe microscopy. ${ }^{3}$ Small apex curvatures facilitate the application of an intense dc electric field in the order of $1-10 \mathrm{GV} / \mathrm{m}$ at the emitter apex with practical voltages to lower the work function substantially by the Schottky effect. Combined with the dc field, electron bunches in the femtosecond time scale can be generated by irradiating the needle cathodes by near infrared femtosecond light pulses. ${ }^{4-7}$ However, the small emission area of the needle cathodes restricts its applicability to certain high current applications such as the rf power amplifiers and the short-wavelength freeelectron lasers.

The field-emitter arrays (FEAs) realized by Spindt ${ }^{8}$ can emit large current by applying low gate bias voltages of $\sim 100 \mathrm{~V}$ or below. Several authors have pointed out the potential advantage of FEAs for the microwave power amplifier applications but the high current operation at frequencies much higher than $\sim 10 \mathrm{GHz}$ has remained to be a challenging task. ${ }^{9-11}$ The high speed switching and photoresponse of silicon FEAs have been explored in detail ${ }^{12-15}$ and the carrier-transport-limited switching down to $\sim 100$ ps was recently reported. ${ }^{15}$ Compared with semiconductor FEAs, metallic FEAs are more robust and potentially more advantageous for the generation of even faster electron bunches with high emission current. However, only a limited number of authors have studied the effect of the laser irradiation on metallic FEAs so far. ${ }^{16}$

In this letter, we investigate the field-emission properties of molybdenum FEAs with single-gate electrodes under the irradiation of intense femtosecond light pulses in near infrared. Despite the small fraction of the electron emission area, we observe a single-photon photoelectric current from the emitter tips with a high quantum efficiency, superposed on the dc field-emission current.

\footnotetext{
${ }^{a)}$ Electronic mail: soichiro.tsujino@psi.ch.
}

The FEA devices (Fig. 1) used for the experiment are fabricated in combination of the molding technique and the self-aligned gate process. The field-emitter and the gate electrode material consist of molybdenum deposited by sputtering at room temperature. The pyramidal-shaped field-emitter structures are aligned in a square or a hexagonal array with 5-10 $\mu \mathrm{m}$ period. The substrate of the emitter structures consists of a $500 \mu \mathrm{m}$ thick electroplated nickel plate. The distance from the emitter tip to the edge of the gate aperture hole is $\sim 1.2 \mu \mathrm{m}$. The apex size of the emitters (inset of Fig. 1) is $\sim 20 \mathrm{~nm}$ in diameter. ${ }^{17}$

In the experiment, we measured several FEA devices having $39 \times 39$ tip square arrays aligned with $5 \mu \mathrm{m}$ period (total array area of $200 \mu \mathrm{m}^{2}$ ). An FEA was mounted on a copper holder and the gate electrode is wire bonded to an insulated electrode. Then the device is loaded in the vacuum chamber which is subsequently evacuated down to the base pressure below $5 \times 10^{-9}$ mbar. A positive bias voltage is applied to the gate electrode in dc while the substrate and the emitter arrays were held at ground potential. The emitted electrons are collected by a cylindrical shape anode with a diameter of $15 \mathrm{~mm}$, placed in front of FEAs with a separa-

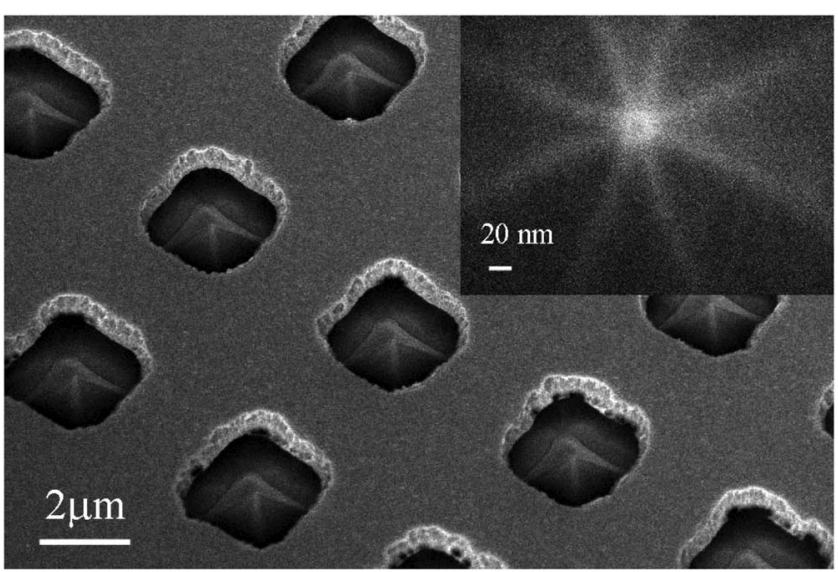

FIG. 1. Scanning electron microscope images of the Mo field-emitters aligned with a $5 \mu \mathrm{m}$ period. The gate electrode is a $0.5 \mu \mathrm{m}$ thick Mo film separated from the emitter substrate by a $1.2 \mu \mathrm{m}$ thick $\mathrm{SiO}_{2}$ film. Individual emitters are $\sim 1.3 \mu \mathrm{m}$ tall. The inset is the apex of an emitter imaged from the top at higher magnification, showing that the apex diameter is equal to $\sim 20 \mathrm{~nm}$. 

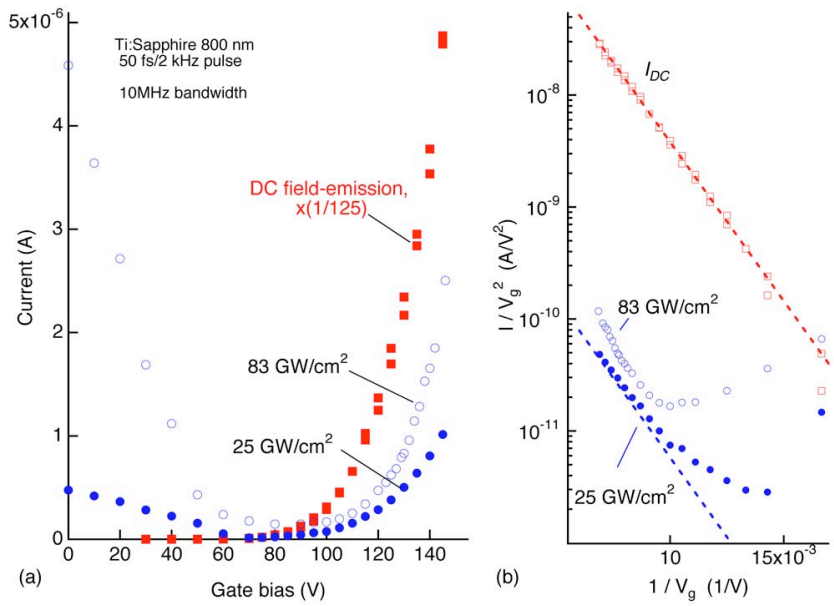

FIG. 2. (Color online) (a) dc field-emission current $I_{\mathrm{dc}}$ (filled squares) and the photoelectric signals vs gate bias voltages when the FEA is irradiated at $25 \mathrm{GW} / \mathrm{cm}^{2}$ (filled circles), and at $83 \mathrm{GW} / \mathrm{cm}^{2}$ (empty circles). (b) The FN plot of $I_{\mathrm{dc}}$ (squares) and of the photoelectric signals at these two laser intensities. The linear slope of the relation between $\ln \left(I_{\mathrm{dc}} / V_{g}^{2}\right)$ and $1 / V_{g}$ suggests that the emitter apex field is given by $\beta V_{g}$ with $\beta$ equal to $(5-10)$ $\times 10^{7} \mathrm{~m}^{-1}$.

tion of $\sim 20 \mathrm{~mm}$. The anode is dc biased at $1 \mathrm{kV}$ while monitoring the dc current. The ac anode current was detected by a load resistor of $99.2 \Omega$, amplified, and monitored by a digital oscilloscope. Most of the data were collected by using a $60 \mathrm{~dB}$ gain voltage amplifier with $10 \mathrm{MHz}$ bandwidth. An amplifier with $2 \mathrm{GHz}$ bandwidth was used optionally. When the gate bias voltage $V_{g}$ is increased above $\sim 50 \mathrm{~V}$, the device starts to emit a dc field-emission current $I_{\mathrm{dc}}$ (Fig. 2) which reaches $0.5-1 \mathrm{~mA}$ when increasing $V_{g}$ to $140-145 \mathrm{~V}$. The relation between $I_{\mathrm{dc}}$ and $V_{g}$ is described well by the empirical Fowler-Nordheim (FN) equation. This is indicated by the linear FN plot in Fig. 2(b), i.e., the relation between $\ln \left(I_{\mathrm{dc}} / V_{g}^{2}\right)$ and $1 / V_{g}$ is approximately linear. ${ }^{18}$ The background pressure increased up to $(1-4) \times 10^{-8}$ mbar when operating the FEA at high emission current due to the electron bombardment of the anode.

The near infrared pulses are generated by a Ti:sapphire chirped pulse amplifier system delivering ultrashort laser pulses of $50 \mathrm{fs}$ duration centered at a wavelength of $800 \mathrm{~nm}$ at a repetition rate of $2 \mathrm{kHz}$. The linearly polarized laser beam is focused onto the sample to a spot size of 320 $\times 690 \mu \mathrm{m}^{2}$ full width at half maximum with an incidence angle of $63^{\circ} \pm 2^{\circ}$ from the surface normal. Approximately $14 \%$ of the pulse energy is coupled to the active $200 \mu \mathrm{m}$ square part of the devices. The pulse energy was kept below $13 \mu \mathrm{J}$ corresponding to the maximum intensity of $94 \mathrm{GW} / \mathrm{cm}^{2}$. This is approximately a factor of 10 below the laser ablation threshold of our devices as determined by a separate experiment.

Figure 3(a) shows the photocurrent pulses at various $V_{g}$ for the excitation light intensity $I_{\mathrm{NIR}}$ of $25 \mathrm{GW} / \mathrm{cm}^{2}$ and with the optical electric field within the incident plane ( $p$ polarization). When increasing $V_{g}$ from 0 to $70 \mathrm{~V}$, the photocurrent decreases by a factor of 34 . When raising $V_{g}$ further, the photocurrent exponentially grows and reaches, at $140 \mathrm{~V}$, a value larger than the zero-bias signal. For $V_{g}$ above $70 \mathrm{~V}$, a slow component of the photocurrents in the order of a few microamperes (about $0.1 \%$ of $I_{\mathrm{dc}}$ ) is also observed. The slow signal decays over $\sim 5 \mu$ s (not shown).
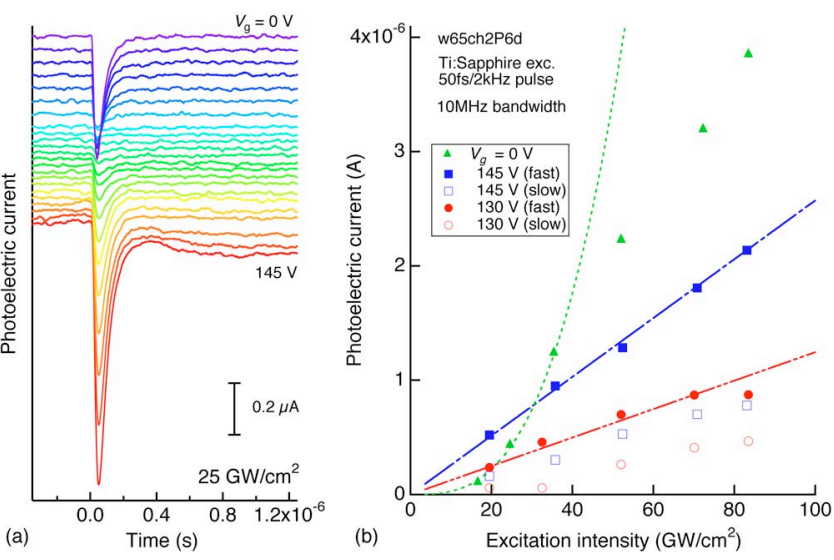

FIG. 3. (Color online) (a) Photoelectric current pulse from the Mo FEA device observed when the gate bias voltages $V_{g}$ was varied from 0 to $145 \mathrm{~V}$, when the device was irradiated by the $p$-polarized $50 \mathrm{fs}$ laser pulses at a peak intensity $I_{\text {NIR }}$ of $25 \mathrm{GW} / \mathrm{cm}^{2}$ (from top to down, $V_{g}$ was varied by $10 \mathrm{~V}$ step between 0 and $70 \mathrm{~V}$ and by $5 \mathrm{~V}$ between 75 and $145 \mathrm{~V}$; the photocurrent was measured using an amplifier with $10 \mathrm{MHz}$ frequency bandwidth). (b) Photoelectric current signal vs laser intensity with $V_{g}$ set to $0 \mathrm{~V}$ (filled triangle), $130 \mathrm{~V}$ (circles), and $145 \mathrm{~V}$ (squares). For the latter, the filled signs represent the fast and the empty symbols the slow signal component. The broken curve shows the $I_{\mathrm{NIR}}^{3}$ dependence of the zero bias signal at low excitation intensity, evidencing the three photon photoemission process which becomes saturated at high excitation. The chain lines are the linear fit of the fast signals for $V_{g}$ equal to $130 \mathrm{~V}$ (double chain) and $145 \mathrm{~V}$ (single chain) indicating that these are generated by single-photon photoemission.

The zero-bias photocurrent is assigned to the threephoton photoemission (3PPE) from the gate electrode that occupies $94 \%$ of the device area. This can be concluded from the pulse energy dependence of the signal (see below). The application of a positive $V_{g}$ creates a potential barrier for the electrons at the surface of the gate electrode, and thus inhibits the 3PPE process. On the other hand, the fast component of the photocurrent observed for a bias above $70 \mathrm{~V}$ is attributed to the field-emission from the nanotip array enhanced by the incident laser field. The concomitant increase of $I_{\mathrm{dc}}$ with the increase of $V_{g}$ is consistent with this interpretation (Fig. 2). Accordingly, the slow signal is ascribed to the increase of the lattice temperature of the emitters by the light absorption, in the order of $10^{\circ}$, and the resultant transient enhancement of $I_{\mathrm{dc}}{ }^{18}$ When the laser polarization is perpendicular to the incident plane ( $s$ polarization), the signal at $V_{g}=130 \mathrm{~V}$ decreases to $65 \%$ of the one obtained with $p$-polarized light. This polarization dependence also supports the above interpretation that we indeed observe the ultrafast electron emission from the emitter tips induced by the laser field. ${ }^{4-7}$

Figure 3(b) shows the dependence of the peak photocurrent on $I_{\text {NIR }}$ for $V_{g}$ equal to 0,130 , and $145 \mathrm{~V}$. The cubic dependence of the zero-bias signal at low laser intensity confirms the 3PPE process. In fact, the three-photon energy of $4.64 \mathrm{eV}$ compares well to the work function of molybdenum of $\sim 4.6 \mathrm{eV} .{ }^{19}$ At large $I_{\mathrm{NIR}}$, the increase of the 3PPE signal becomes saturated, which is similar to previous reports. ${ }^{20} \mathrm{In}$ contrast, the $I_{\mathrm{NIR}}$ dependence at two finite bias voltages varies as $I_{\mathrm{NIR}}^{(1+\varepsilon)}$ with $\varepsilon$ equal to $0.01 \pm 0.04$ and $0.08 \pm 0.09$ for $V_{g}$ equal to 130 and $145 \mathrm{~V}$, respectively. Hence, within the experimental uncertainty, the photocurrent at these conditions is generated by single-photon photoemission. The large dc electric field $F_{\mathrm{dc}}$ at the emitter apex and resultant lowering of the work function are the keys to observe the near infrared 
single-photon photoemission from the field-emitter structures. Due to the Schottky effect, the work function of the molybdenum is lower than the single photon energy when $F_{\mathrm{dc}}$ is larger than $6 \mathrm{GV} / \mathrm{m}$. In fact, by evaluating the relation between $F_{\mathrm{dc}}$ and $V_{g}$ from the slope of the FN plot, ${ }^{18}$ we found that, when $V_{g}$ was equal to $145 \mathrm{~V}, F_{\mathrm{dc}}$ was equal to $7-14 \mathrm{GV} / \mathrm{m}^{21}$

The actual pulse width of the fast current component is likely to be in the order of the light pulse width and is limited here by the bandwidth of the measurement electronics. ${ }^{22}$ Therefore, the peak current amplitude is proportional to the emission charge but the quantification of the value requires a separate calibration. This is done by measuring the photocurrent using a dc ammeter at zero $V_{g}$ when $I_{\mathrm{dc}}$ was zero and comparing it to the pulsed peak current amplitude. From this calibration, we found the maximum single-photon quantum efficiency $\eta_{\text {ext }}$ of $2.1 \times 10^{-7}$. This was observed at the largest $V_{g}$ and corresponds to the maximum charge of the fast component of the electronic bunch of $0.27 \mathrm{pC}$. Assuming that the laser-induced electron bunch has the same pulse width as the excitation pulse and considering the spatial chirp of maximum 600 fs across the $200 \mu \mathrm{m}$ device diameter due to the oblique angle of incidence, ${ }^{23}$ the measured charge corresponds to a peak current of $0.5 \mathrm{~A}$. The bunch length and the peak current can be improved by appropriate compensation. ${ }^{23,24}$

Finally, we note that $\eta_{\mathrm{ext}}$ can be written as $(1-R)$ $\eta_{\text {tip }}(r / p)^{2}$ where $R=0.56$ is the reflectance of the molybdenum at $800 \mathrm{~nm}, \eta_{\text {tip }}$ is the quantum efficiency at the emitter apex, and $(r / p)^{2}$ is the fraction of the emitter tip area given by the emitter diameter $r$ equal to $\sim 20 \mathrm{~nm}$ and the emitter period $p$ equal to $5 \mu \mathrm{m}$. From these values, we found that $\eta_{\text {tip }}$ is equal to $\sim 3 \times 10^{-2}$. A quantum efficiency for near infrared radiation of the similar order of magnitude was also observed in low work function materials such as the dispenser photocathode. ${ }^{25}$ From the linearity of the signal, it is likely that the laser-induced emission charge from the FEAs can be significantly increased at the larger light pulse energy when the pulse width is proportionally increased with the pulse energy. The upscaling of the emission charge, the characterization of the emittance, and the elucidation of the electron emission mechanism are the subjects of further study.

In summary, we demonstrated the single-photon photoelectric current from molybdenum FEAs induced by near infrared laser pulses with 50 fs pulse duration. Despite the small fraction of the emission area from the field-emitter tips, up to $\sim 0.3 \mathrm{pC}$ of the emission charge from emitter tips was observed with the external quantum efficiency of $\sim 2$ $\times 10^{-7}$. Our result indicates that metallic FEAs are promising for applications that require high-brightness ultrashort electron beams.

${ }^{1}$ A. H. Zewail, Annu. Rev. Phys. Chem. 57, 65 (2006).

${ }^{2}$ C. A. Brau, Nucl. Instrum. Methods Phys. Res. A 407, 1 (1998).

${ }^{3}$ St. Grafström, J. Appl. Phys. 91, 1717 (2002).

${ }^{4}$ P. Hommelhoff, Y. Sortais, A. Aghjani-Talesh, and M. A. Kasevich, Phys. Rev. Lett. 96, 077401 (2006).

${ }^{5}$ P. Hommelhoff, C. Kealhofer, and M. A. Kasevich, Phys. Rev. Lett. 97, 247402 (2006).

${ }^{6}$ C. Ropers, D. R. Solli, C. P. Schulz, C. Lienau, and T. Elsaesser, Phys. Rev. Lett. 98, 043907 (2007).

${ }^{7}$ B. Barwick, C. Corder, J. Strohaber, N. Chandler-Smith, C. Uiterwaal, and H. Batellan, New J. Phys. 9, 142 (2007).

${ }^{8}$ C. A. Spindt, J. Appl. Phys. 39, 3504 (1968).

${ }^{9}$ P. M. Lally, Y. Goren, and E. A. Nettesheim, IEEE Trans. Electron Devices 36, 2738 (1989).

${ }^{10}$ J. P. Calame, H. F. Gray, and J. L. Shaw, J. Appl. Phys. 73, 1485 (1993).

${ }^{11}$ D. R. Whaley, B. M. Gannon, W. O. Heinen, K. E. Kreischer, C. E. Holland, and C. A. Spindt, IEEE Trans. Plasma Sci. 30, 998 (2002).

${ }^{12}$ H. Ishizuka, Y. Kawamura, K. Yokoo, H. Mimura, H. Shimawaki, and A. Hosono, Nucl. Instrum. Methods Phys. Res. A 483, 305 (2002).

${ }^{13}$ H. Mimura, T. Ukeda, H. Shimawaki, and K. Yokoo, J. Vac. Sci. Technol. B B22, 1218 (2004).

${ }^{14}$ K. X. Liu, C.-J. Chiang, and J. P. Heritage, J. Appl. Phys. 99, 034502 (2006).

${ }^{15}$ C.-J. Chiang, K. X. Liu, and J. P. Heritage, Appl. Phys. Lett. 90, 083506 (2007).

${ }^{16}$ O. Yavas, N. Suzuki, M. Takai, A. Hosono, and S. Kawabuchi, Appl. Phys. Lett. 72, 2797 (1998).

${ }^{17}$ E. Kirk, S. Tsujino, T. Vogel, H. Sehr, and J. Gobrecht, A. Wrulich (unpublished).

${ }^{18}$ C. A. Spindt, I. Brodie, L. Humphrey, and E. R. Westerberg, J. Appl. Phys. 47, 5248 (1976).

${ }^{19}$ CRC Handbook of Chemistry and Physics, 79th ed., edited by D. R. Lide (CRC Press LLC, Boca Raton, 1998), pp. 12-124.

${ }^{20}$ J. G. Fujimoto, J. M. Liu, E. P. Ippen, and N. Bloembergen, Phys. Rev. Lett. 53, 1837 (1984).

${ }^{21}$ The estimation error of $F_{\mathrm{dc}}$ arises from the uncertainty of the slope of the FN-plot. It varied in time and on the $V_{g}$-scan directions as well. However, after an extensive conditioning procedure, the slope became stable within a factor of two. Similarly to Ref. 18, the emitter apex diameter estimated from the fitting of the FN-plot was smaller than the geometrical area obtained from SEM by a factor of 5-10.

${ }^{22}$ From an additional experiment with $2 \mathrm{GHz}$-amplifier, we found that the fast current pulse was shorter than $0.8 \mathrm{~ns}$, which was still limited by the bandwidth of the amplifier.

${ }^{23}$ P. Baum and A. H. Zewail, Proc. Natl. Acad. Sci. U.S.A. 103, 16105 (2006).

${ }^{24}$ Zs. Bor and B. Racz, Opt. Commun. 54, 165 (1985).

${ }^{25}$ N. A. Moody, K. Jensen, D. W. Feldman, P. G. O'Shea, and E. J. Montgomery, Appl. Phys. Lett. 90, 114108 (2007). 